\title{
ON THE ISOPERIMETRIC PROBLEM FOR THE LAPLACIAN WITH ROBIN AND WENTZELL BOUNDARY CONDITIONS
}

\author{
JAMES B. KENNEDY
}

(Received 16 April 2010)

2000 Mathematics subject classification: primary 35P15; secondary 35J05, 35B40, 35J20, 35J25, 35K15, 47A07, 47A10, 47D06, 47F05.

Keywords and phrases: isoperimetric problem, Laplacian, Robin boundary conditions, Wentzell boundary conditions.

We consider the eigenvalues of the Laplacian $-\Delta u=\lambda u$ in a bounded domain $\Omega \subset \mathbb{R}^{N}, N \geq 2$, equipped with Robin boundary conditions

$$
\frac{\partial u}{\partial v}+\alpha u=0
$$

or generalized Wentzell boundary conditions

$$
\Delta u+\beta \frac{\partial u}{\partial v}+\gamma u=0
$$

on $\partial \Omega$, where $v$ is the outer unit normal to $\partial \Omega$, and for us $\alpha, \beta, \gamma$ are nonzero constants. In this context, isoperimetric problems, also known as shape optimization problems, involve minimizing (or maximizing) a given eigenvalue with respect to the domain $\Omega$ (assumed to have a fixed volume); see, for example, the survey article of Payne [11].

The classical Faber-Krahn inequality states that the first eigenvalue of the Dirichlet Laplacian is smallest when $\Omega$ is a ball. This was recently extended to the Robin case when $\alpha>0$ by Daners [2]. Our first result is that in this case the ball is the unique domain with this property, at least amongst all domains of class $C^{2}$. The method of proof uses a functional of the level sets of the first eigenfunction, together with some tools from geometric measure theory, to estimate the first eigenvalue from below. This is combined with a rearrangement of the ball's eigenfunction onto the domain $\Omega$ and the usual isoperimetric inequality.

Thesis submitted to The University of Sydney, November 2009. Degree approved, March 2010. Supervisor: Dr Daniel Daners; Associate Supervisor: Professor E. N. Dancer.

(C) 2010 Australian Mathematical Publishing Association Inc. 0004-9727/2010 \$16.00 
For the second Robin eigenvalue, we prove that the unique minimizing domain when $\alpha>0$ is the disjoint union of two equal balls (which is again the same as in the Dirichlet case), and set the proof up so it works for the Robin $p$-Laplacian. For the higher eigenvalues, we show that it is in general impossible for a minimizer to exist independently of $\alpha>0$. This is done by considering perturbations of the corresponding Dirichlet and Neumann problems.

When $\alpha<0$, we prove that every eigenvalue behaves like $-\alpha^{2}$ as $\alpha \rightarrow-\infty$, provided only that $\Omega$ is bounded with $C^{1}$ boundary. This extends a result of Lou and Zhu [10] for the first eigenvalue. Our proof involves constructing an explicit test function to use in the minimax formula for the $n$th eigenvalue.

For the Wentzell problem, we prove (or, in most cases, re-prove) general operator properties, including those in the less-studied case $\beta<0$, where the problem is illposed in some sense. (This contrasts with the well-behaved case $\beta>0$.) In particular, when $\beta<0$, we give a new proof of the compactness of the resolvent and the structure of the spectrum, at least if $\partial \Omega$ is smooth. This is based on the operator matrix approach of Engel [5] for $\beta>0$, which de-couples the problem into a Dirichlet-type operator acting in the interior of the domain, and a Dirichlet-to-Neumann action on the boundary of the domain.

We prove Faber-Krahn-type inequalities in the general case $\beta, \gamma \neq 0$. These are based on their Robin counterparts, via the elementary trick of identifying every Wentzell eigenvalue as that of a suitable Robin problem, together with a type of fixed point argument. In the 'best' case $\beta, \gamma>0$ we exploit this further to establish a type of equivalence property between the Wentzell and Robin minimizers for all eigenvalues. In particular, this yields a minimizer of the second Wentzell eigenvalue. Finally, we also prove a Cheeger-type inequality for the first eigenvalue in this case (see $[1,6]$ ).

The new material in this thesis has been published in [3, 4, 7-9]. The thesis itself is available online at http://hdl.handle.net/2123/5972.

\section{References}

[1] J. Cheeger, 'A lower bound for the smallest eigenvalue of the Laplacian', in: Problems in Analysis (Papers dedicated to Salomon Bochner, 1969) (Princeton University Press, Princeton, NJ, 1970), pp. 195-199.

[2] D. Daners, 'A Faber-Krahn inequality for Robin problems in any space dimension', Math. Ann. 335(4) (2006), 767-785.

[3] D. Daners and J. Kennedy, 'Uniqueness in the Faber-Krahn inequality for Robin problems', SIAM J. Math. Anal. 39(4) (2007/08), 1191-1207.

[4] D. Daners and J. B. Kennedy, 'On the asymptotic behaviour of the eigenvalues of a Robin problem with a large parameter', Differential Integral Equations 23(7-8) (2010), 659-669.

[5] K.-J. Engel, 'The Laplacian on $C(\bar{\Omega})$ with generalized Wentzell boundary conditions', Arch. Math. (Basel) 81(5) (2003), 548-558.

[6] B. Kawohl and V. Fridman, 'Isoperimetric estimates for the first eigenvalue of the $p$-Laplace operator and the Cheeger constant', Comment. Math. Univ. Carolin. 44(4) (2003), 659-667.

[7] J. Kennedy, 'A Faber-Krahn inequality for the Laplacian with generalised Wentzell boundary conditions', J. Evol. Equ. 8(3) (2008), 557-582. 
[8] J. Kennedy, 'An isoperimetric inequality for the second eigenvalue of the Laplacian with Robin boundary conditions', Proc. Amer. Math. Soc. 137(2) (2009), 627-633.

[9] J. B. Kennedy, 'On the isoperimetric problem for the higher eigenvalues of the Robin and Wentzell Laplacians', Z. Angew. Math. Phys., to appear. Preprint, arXiv:0910.3966v1.

[10] Y. Lou and M. Zhu, 'A singularly perturbed linear eigenvalue problem in $C^{1}$ domains', Pacific J. Math. 214(2) (2004), 323-334.

[11] L. E. Payne, 'Isoperimetric inequalities and their applications', SIAM Rev. 9 (1967), 453-488.

JAMES B. KENNEDY, Group of Mathematical Physics,

University of Lisbon, Av. Prof. Gama Pinto 2, 1649-003 Lisboa, Portugal

e-mail: jkennedy@cii.fc.ul.pt 